\title{
Erastin induces apoptotic and ferroptotic cell death by inducing ROS accumulation by causing mitochondrial dysfunction in gastric cancer cell HGC-27
}

\author{
YINGWEI SUN $^{1 *}$, RONGRONG DENG ${ }^{2 *}$ and CUIWEI ZHANG ${ }^{3}$ \\ ${ }^{1}$ Department of Pharmacy, The Teaching Hospital of Chengdu University of Traditional Chinese Medicine; \\ ${ }^{2}$ Medicine School of Medical and Life Sciences, Chengdu University of Traditional Chinese Medicine, \\ Chengdu, Sichuan 610072; ${ }^{3}$ Department of Pathology, The Affiliated Hospital of \\ Southwest Medical University, Luzhou, Sichuan 646000, P.R. China
}

Received January 22, 2020; Accepted May 27, 2020

DOI: $10.3892 / \mathrm{mmr} .2020 .11376$

\begin{abstract}
Erastin, a classical inducer of non-apoptotic cell death, exerts cytotoxicity in several types of cancer cells, including gastric cancer cells, by depleting glutathione, which is a primary cellular antioxidant, thus causing reactive oxygen species (ROS) accumulation. Although numerous studies have focused on the non-apoptotic cell death induced by erastin, whether erastin induces apoptosis remains unknown. The present study confirmed the cytotoxicity of erastin in HGC-27 cells and used a $30 \%$ inhibitory concentration $\left(\mathrm{IC}_{30}\right.$, approximately $6.23 \mu \mathrm{M}$ ) for further analysis. The cell cycle analysis revealed that $6.23 \mu \mathrm{M}$ of erastin inhibited proliferation by blocking the cell cycle at the G1/G0 phase. Further analysis also showed that $6.23 \mu \mathrm{M}$ of erastin clearly inhibited HGC-27 malignant behaviors, including migration, invasion, colony formation and tumor formation in soft agar. The observation of ROS accumulation due to erastin treatment led to determination of the effects of erastin on mitochondrial function and, as expected, erastin treatment decreased transcriptional activity and ATP production in mitochondria and disrupted the mitochondrial potential; these effects were reversed by the addition of the ROS scavenger NAC. To evaluate the effect of erastin in inducing apoptosis, HGC-27 cells were treated with $6.23 \mu \mathrm{M}$ of erastin for 7 days and then analyzed. Evident apoptotic cell death was induced by erastin and this apoptosis was reversed by the addition of an apoptosis inhibitor (zVAD) or NAC but not by the addition of a ferroptosis inhibitor
\end{abstract}

Correspondence to: Dr Cuiwei Zhang, Department of Pathology, The Affiliated Hospital of Southwest Medical University, 25 Taiping Street, Luzhou, Sichuan 646000, P.R. China

E-mail: zhangcuiwei_smu@163.com

*Contributed equally

Key words: apoptosis, stomach neoplasms, reactive oxygen species, gastric cancer cells, mitochondria (ferrostatin-1). Furthermore, the detection of caspase-3 and poly (adenosine diphosphate-ribose) polymerase (PARP) also confirmed that treatment with erastin promoted the cleavage of caspase-3 and PARP, which are hallmarks of apoptosis. Taken together, the present study revealed that a low dose of erastin inhibited malignant behavior and induced apoptosis by causing mitochondrial dysfunction.

\section{Introduction}

Erastin is a classic ferroptosis inducer that suppresses the glutamate/cystine antiporter (system $\mathrm{X}_{\mathrm{C}}{ }^{-}$), subsequently inhibits cellular cystine uptake and depletes glutathione (GSH) (1). GSH is a primary cellular antioxidant that serves critical functions in maintaining the redox balance and defending against oxidative stress, including reactive oxygen species (ROS) (1). Erastin has been widely found to induce ferroptosis in several types of cancer cells. Yang et al (2) found that in fibrosarcoma, a fatal dose of erastin induces ferroptosis by depleting $\mathrm{GSH}$, confirmed to be an important target of erastin. DeHart et al (3) found that in the hepatocarcinoma cell lines HepG2 and Huh7, erastin binds to voltage-dependent anion channels and thus increases $\Delta \Psi$, leading to mitochondrial ROS generation followed by ferroptosis induction. Pan et al (4) reported that in non-small cell lung cancer cells, erastin can induce ferroptosis, which can be reversed by ferrostatin-1, a ferroptosis inhibitor, but not by Z-VAD-FMK, an apoptosis inhibitor, indicating that erastin-induced cell death is a non-apoptotic cell death.

ROS are a heterogeneous group of highly reactive ions and molecules that are derived from molecular oxygen (5). ROS are believed to act as an antioxidant system that is critical for maintaining redox homeostasis (6) and to be toxic and closely associated with various pathological mechanisms (7). The dual roles of ROS can be briefly described as follows: A relatively low or controlled increase in ROS regulates cell proliferation, apoptosis, angiogenesis and other physiological processes (5), and an abnormal generation or accumulation of ROS can induce oxidative stress and damage programmed physiological processes by damaging cellular lipids, proteins and DNA (8). Thus, the balance of ROS is critical for regulating physiological 
processes or predicting oxidative stress. Considering the ROS accumulation caused by erastin, the present study hypothesized that erastin potentially regulated physiological processes by affecting the balance of ROS levels.

Gastric cancer has been the second leading cause of cancer-related mortality worldwide in recent decades (9). Numerous therapeutic strategies have been developed, but most patients are asymptomatic in the early stages and more than half of the patients suffer from distant metastasis; therefore, gastric cancer has a poor prognosis and the 5-year survival rate is $<10 \%(10,11)$. Thus, substantial effort has been made to determine the mechanisms of gastric carcinogenesis. By considering the relatively high levels of ROS in cancer cells compared with normal cells, cancer cells are believed to be more sensitive to oxidative stress and this sensitivity has become an important therapeutic target for cancer treatment, including treatment of gastric cancer (12). Chen et al (12) revealed that in gastric cancer, the stimulation of ROS by oxidative stress activates the pro-apoptotic pathway and this was hypothesized to be a novel therapeutic strategy for the treatment of gastric cancer. Thus, it is worth determining whether erastin-induced ROS contribute to the physiological processes of gastric cancer cells.

The present study examined the effects of a relatively low dose of erastin on the malignant behaviors of HGC-27 gastric cancer cells, including proliferation, migration, invasion, colony formation and soft agar tumor formation. It shows that erastin-induced ROS disrupted mitochondrial function. Together, these results suggest that erastin-induced ROS may contribute to antitumor effects and provide an effective alternative for gastric cancer therapy.

\section{Materials and methods}

Cell culture and treatment. Human gastric cancer cells HGC-27 were obtained from the Institute of Biochemistry and Cell Biology, Chinese Academy of Sciences. Cells were maintained in RPMI-1640, supplemented with $10 \%$ fetal bovine serum (FBS) at $37^{\circ} \mathrm{C}$ and $5 \% \mathrm{CO}_{2}$ humidified incubator. All reagents were purchased from Thermo Fisher Scientific, Inc.

For cell treatment, $0-50 \mu \mathrm{M}$ of erastin was supplemented to the original medium and cell viability was analyzed after $24 \mathrm{~h}$. For apoptosis analysis, the cells were treated with $6.23 \mu \mathrm{M}$ of erastin for 7 days followed by further analysis. To abolish ROS, $10 \mu \mathrm{M}$ NAC was supplemented in original medium; to inhibit ferroptosis, $10 \mu \mathrm{M}$ of ferrostatin-1 was employed; to inhibit apoptosis, $10 \mu \mathrm{M}$ of zVAD was employed.

CCK-8 assay. Cell viability was measured using CCK-8 assay. The cells were plated at a density of $5 \times 10^{3}$ cells per well in 96-well microtiter culture plates, incubated overnight at $37^{\circ} \mathrm{C}$ and treated with specified concentrations of erastin for $24 \mathrm{~h}$. Then, $10 \mu \mathrm{l}$ of CCK-8 was added into each well for another $4 \mathrm{~h}$ incubation and scanned by a multi-spectrophotometer (Thermo Fisher Scientific, Inc.) at $470 \mathrm{~nm}$.

5-Ethynyl-20-deoxyuridine (EdU) staining. Cell proliferation was detected by the incorporation of EdU with an EdU Cell Proliferation Assay kit (Guangzhou RiboBio Co., Ltd.). Briefly, cells were incubated with $50 \mathrm{mM}$ EdU for $4 \mathrm{~h}$ and then fixed with $4 \%$ paraformaldehyde for $30 \mathrm{~min}$ at $25^{\circ} \mathrm{C}$. Then, EdU staining was performed according to the manufacturer's protocol. Cell nuclei were stained with Hoechst-33342 (Sigma-Aldrich; Merck KGaA) at a concentration of $1 \mathrm{mg} / \mathrm{ml}$ for $20 \mathrm{~min}$. The proportion of cells incorporating EdU was determined using an X71 (U-RFL-T) fluorescence microscope (Olympus Corporation; magnification, $\mathrm{x} 40$ ).

Propidium iodide (PI) staining followed by flow cytometry. The cells were trypsinized and suspended in ice-cold PBS. After centrifugation ( $200 \mathrm{x} \mathrm{g}$ ) for $5 \mathrm{~min}$ at room temperature, the cells were fixed with ice-cold $70 \%$ ethanol. After $4 \mathrm{~h}$, the cells were pelleted by centrifugation $(200 \mathrm{x}$ g) for $5 \mathrm{~min}$ at room temperature and washed with ice-cold PBS and suspended with $5 \mu \mathrm{g} / \mathrm{ml}$ PI (Sigma-Aldrich; Merck KGaA) and $10 \mu \mathrm{g} / \mathrm{ml}$ RNase A (Sigma-Aldrich; Merck KGaA) for 10 min in darkness. Then, the stained cells were pelleted by centrifugation ( $200 \mathrm{x} \mathrm{g}$ ) for $5 \mathrm{~min}$ at room temperature and washed with three washes using ice-cold PBS and then analyzed using three laser Navios flow cytometers (Beckman Coulter, Inc.) according to the manufacturer's instructions.

Cell migration and invasion assay. Cells were seeded in a 6 -well plate at a density of $2.5 \times 10^{5}$ cells/well. A scratch was made with a sterile $10-\mu \mathrm{l}$ pipette tip. After 0 and $24 \mathrm{~h}$, images were captured.

To measure invasion ability, $5 \times 10^{3}$ cells were suspended and plated into upper chamber $(8-\mu \mathrm{m}$ pore size; Corning Inc.) coated with Matrigel for $24 \mathrm{~h}$ at $37^{\circ} \mathrm{C}$ (Sigma-Aldrich; Merck $\mathrm{KGaA})$. Then, the chamber was fixed with $4 \%$ paraformaldehyde at $25^{\circ} \mathrm{C}$ for $20 \mathrm{~min}$ and stained with $0.1 \%$ crystal violet for $20 \mathrm{~min}$ at $25^{\circ} \mathrm{C}$ (Sigma-Aldrich).

Colony formation. The cells were trypsinized and suspended in PBS. Subsequently, 1,000 cells were seeded in 6-well plates and cultured for 2-3 weeks. When the colonies were visible to the naked eye, the plate was washed twice with PBS. The colonies were fixed with $4 \%$ paraformaldehyde for $10 \mathrm{~min}$ and stained by $0.25 \%$ crystal violet for $20 \mathrm{~min}$ at $25^{\circ} \mathrm{C}$.

Tumor formation in soft agar. Cells were resuspended with $0.3 \%$ soft agar in RPMI-1640 containing 10\% FBS, then layered onto $0.6 \%$ solidified agar in RPMI-1640 containing 10\% FBS in 6-well plates $\left(5 \times 10^{3}\right.$ cells/well). These plates were incubated at $37^{\circ} \mathrm{C}$ for 2-3 weeks. Colonies containing $\geq 50$ cells were counted using Image J software (version-2.0; National Institutes of Health).

ROS detection. The specific fluorescent probe $\mathrm{H}_{2} \mathrm{DCFDA}$ (Sigma-Aldrich) was used to detect the intracellular ROS level. $\mathrm{H}_{2}$ DCFDA (5 $\left.\mu \mathrm{l}\right)$ was added for $15 \mathrm{~min}$ at $25^{\circ} \mathrm{C}$. The cells in 6 -well plates were washed three times with PBS and imaged using an X71 (U-RFL-T) fluorescence microscope (Olympus Corporation, magnification, $\mathrm{x} 40$ ).

Mitochondrial transcriptional activity. RNA was isolated from cells following the manufacturer's instructions using RNeasy kit (Qiagen $\mathrm{GmbH}$ ). Reverse transcription was performed using a high capacity RNA-to-cDNA kit (Thermo Fisher Scientific, Inc.). Quantitative PCR was performed using SYBR Green PCR Master Mix (Thermo 
Fisher Scientific, Inc.) and the primers were as follows: COXI: Forward, 5'-ATGCGGCCATAGGTTCTGC-3' and reverse, 5'-TCCTCAAGATGTCTCAGTTCCAT-3'; ND1: Forward, 5'-TCGTCATAATCTGTCCCTACACA-3' and reverse, 5'-CGGCTTCGGCTCTTAGCAAA-3'; and $\beta$-actin: Forward, 5'-GTGACGTTGACATCCGTAAAG A-3' and reverse, 5'-GCCGGACTCATCGTACTCC-3'. The thermocycling conditions were as follows: $95^{\circ} \mathrm{C}$ for $5 \mathrm{~min}$, 40 cycles of $95^{\circ} \mathrm{C}$ for $10 \mathrm{sec}, 60^{\circ} \mathrm{C}$ for $50 \mathrm{sec}$. The amplification and analysis were performed using an ABI Prism 7500 Real-Time PCR System (Applied Biosystems; Thermo Fisher Scientific, Inc.). Samples were normalized to a housekeeping gene using the $2^{-\Delta \Delta \mathrm{Cq}}$ method (13).

ATP measurement. Cells $\left(1 \times 10^{6}\right)$ were suspended and washed with ice-cold PBS. Cells pelleted by centrifugation $(200 \mathrm{x} \mathrm{g})$ for $5 \mathrm{~min}$ at room temperature were suspended in ATP measuring buffer and ATP level was measured using the ATP Bioluminescence Assay kit (Roche Diagnostics) following the manufacturer's instructions.

JC-1 staining. JC-1 probe (Sigma-Aldrich; Merck KGaA) was used to investigate the mitochondrial membrane potential following erastin treatment. HGC-27 cells were incubated with JC-1 $(20 \mu \mathrm{g} / \mathrm{ml})$ at $37^{\circ} \mathrm{C}$ for $20 \mathrm{~min}$ and then washed twice with PBS. Treated cells were observed by an X71 (U-RFL-T) fluorescence microscope (Olympus Corporation, magnification, $\mathrm{x} 40$ ).

Carboxyfluorescein diacetate, succinimidyl ester (CFSE)/PI double staining. Cells $\left(5 \times 10^{5}\right)$ were seeded in a 6 -well plate at a density of $2.5 \times 10^{5}$ cells/well, suspended and washed with ice-cold PBS and stained with $10 \mu \mathrm{M} \mathrm{CFSE}$ at $37^{\circ} \mathrm{C}$ for $2 \mathrm{~h}$. Then cells were seeded in 12-well plates and treated with erastin. After $24 \mathrm{~h}$, the medium was removed and the cells were washed with ice-cold PBS twice and incubated with $5 \mu \mathrm{g} / \mathrm{ml} \mathrm{PI}$ for $10 \mathrm{~min}$ at $25^{\circ} \mathrm{C}$ in dark. Then the supernatant was removed and cells were imaged using an X71 (U-RFL-T) fluorescence microscope (Olympus Corporation, magnification, $\mathrm{x} 40$ ).

Annexin V/PI double staining. Apoptosis was measured using Annexin V-FITC/PI apoptosis detection kit (BD Biosciences) according to the manufacturer's instructions. The cells were washed with ice-cold PBS and pelleted by centrifugation ( $200 \mathrm{x} \mathrm{g}$ ) for $5 \mathrm{~min}$ at room temperature. Following removal of the supernatant, the pellet was resuspended in $1 \mathrm{X}$ binding buffer and stained with $5 \mu$ l fluorescein isothiocyanate-labeled Annexin $\mathrm{V}$ at $4^{\circ} \mathrm{C}$ for 15 min in the dark. Then $10 \mu \mathrm{l}$ of PI was added at $4^{\circ} \mathrm{C}$ for $5 \mathrm{~min}$ in the dark. The cells were analyzed by flow cytometry using 3 laser Navios flow cytometers (Beckman Coulter, Inc.).

Western blot analysis. The cells suspended in RIPA buffer (Sigma-Aldrich; Merck KGaA) and lysed using SoniConvert ${ }^{\circledR}$ Sonicator (DocSense) and protein concentration was determined via BCA assay (Sigma-Aldrich; Merck KGaA) according to the manufacturer's instruction. In each lane, $20 \mu \mathrm{g}$ total protein was applied to a $10 \%$ SDS-PAGE gel. Fractionated proteins were transferred onto a nitrocellulose membrane, and blocked using 5\% BSA containing PBS at room temperature for $1 \mathrm{~h}$. The membrane was incubated with the primary antibody specific to cleaved PARP, cleaved caspase-3 (1:1,000; cat. no. ab136812; Abcam), at $4^{\circ} \mathrm{C}$ overnight, then incubated with the secondary antibody goat anti-mouse IgG H\&L antibody (HRP-labeled; 1:10,000; ab97040; Abcam) at $37^{\circ} \mathrm{C}$ for $1 \mathrm{~h}$. Mouse anti- $\beta$-actin (1:5,000; cat. no. ab8226; Abcam) was used as an internal reference. Then blots were visualized with chemiluminescence kit (Thermo Scientific, Inc.) and quantitatively measured using Image J software (version-2.0; National Institutes of Health).

Statistical analysis. All experiments were repeated three times independently. The Student's t-test was used to determine the statistical significance between two groups. One-way analysis of variance was performed to compare multiple groups followed by Tukey's post-hoc test. Data in the present study were presented as the mean \pm standard error of the mean. $\mathrm{P}<0.05$ was considered to indicate a statistically significant difference.

\section{Results}

Relative low dose of erastin inhibits cell proliferation in gastric cancer cells HGC-27. To investigate the cellular cytotoxicity of erastin on gastric cancer cells HGC-27, HGC-27 cells were treated with DMOS (Mock) or erastin at different concentration. As shown in Fig. 1A, the 30\% inhibitory concentration $\left(\mathrm{IC}_{30}\right)$ of erastin was $6.23 \pm 0.09 \mu \mathrm{M}$ and the $50 \%$ inhibitory concentration $\left(\mathrm{IC}_{50}\right)$ of erastin was $14.39 \pm 0.38 \mu \mathrm{M}$. To evaluate the effects of relative low concentration of erastin, $6.23 \mu \mathrm{M}$ of erastin was added in HGC-27 culture medium for 5 days and cell viability was measured each day. The decreased cell viability from 1-5 days was confirmed (Fig. 1B). To further confirm the effect of erastin on cell proliferation, EdU staining was performed after erastin treatment for $24 \mathrm{~h}$ and fewer positively stained cells were observed in erastin-treated group (Fig. 1C). Then the cell cycle phases were also analyzed; $6.23 \mu \mathrm{M}$ of erastin treatment increased the proportion of G1/G0 phase and the decreased proportion of S phase in HGC-27 cells, which demonstrated that a relatively low dose of erastin inhibited proliferation via block cell cycle at G1/G0 (Fig. 1D).

Amount of $6.23 \mu \mathrm{M}$ of erastin inhibits malignant behaviors in HGC-27 cells. The present study also confirmed the effects of $6.23 \mu \mathrm{M}$ of erastin on malignant behaviors in HGC-27 cells, including migration, invasion, colony formation and tumor formation in soft agar. In scratch assay, after 24-h healing, the gap in the erastin-treated group was $66.1 \pm 2.4 \mu \mathrm{m}$, which was larger than that in the mock group $(50.3 \pm 1.5 \mu \mathrm{m}$; Fig. $2 \mathrm{~A})$. It was also observed that $6.23 \mu \mathrm{M}$ of erastin treatment inhibited invasion, colony formation and tumor formation (Fig. 2B-D), without knowing whether it was associated with the proliferation inhibition caused by erastin treatment.

Amount of $6.23 \mu \mathrm{M}$ of erastin depolarizes mitochondrial potential and leads to mitochondrial dysfunction. By considering the ROS accumulation effect of erastin treatment, it was then investigated whether $6.23 \mu \mathrm{M}$ of erastin could also induce ROS accumulation. As shown in Fig. 3A, after ROS probe staining using $\mathrm{H}_{2}$ DCFDA, erastin treatment induced an evident 

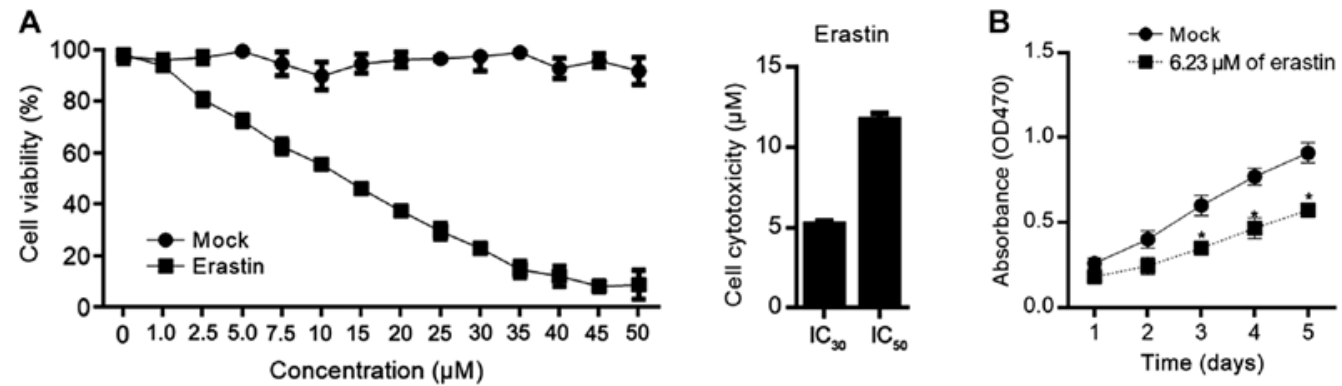

C
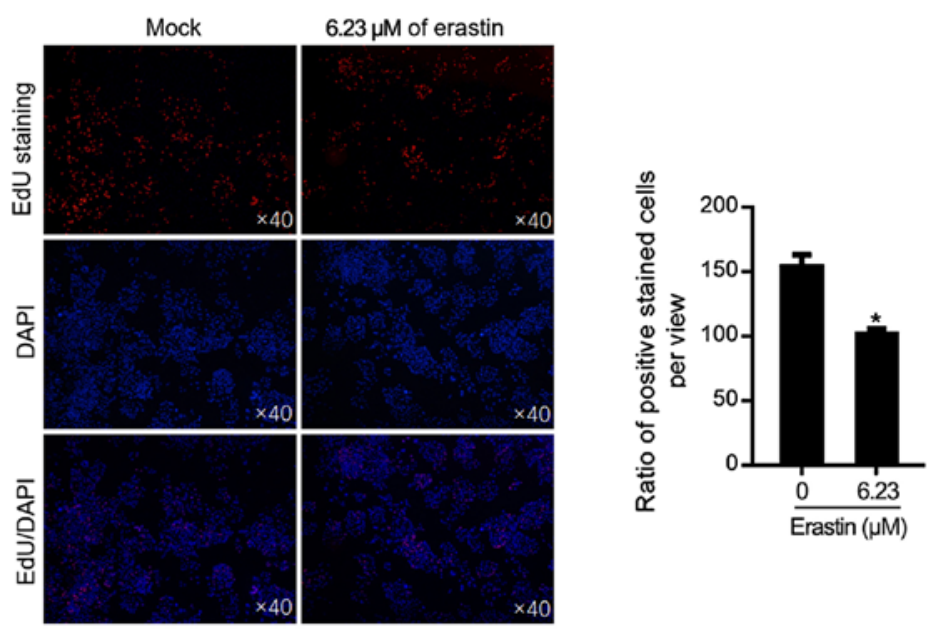

D
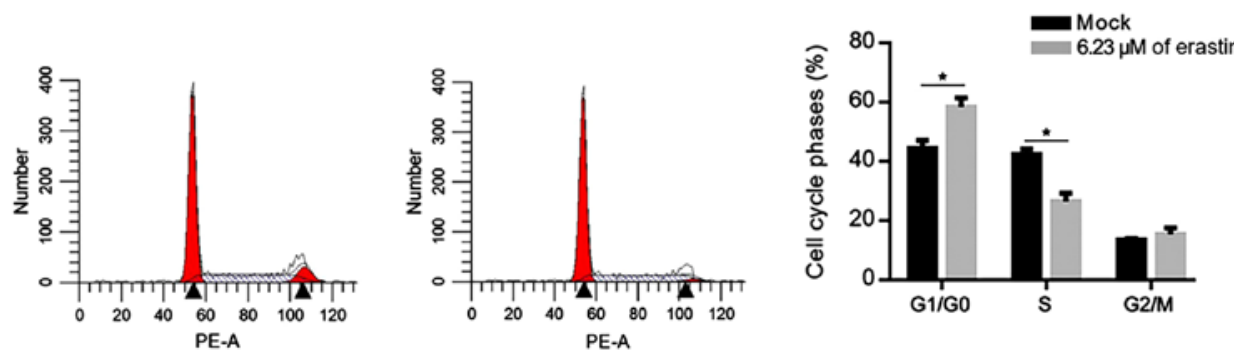

Figure 1. Identification of cytotoxicity of erastin and its effect on proliferation of gastric cancer cells. (A) CCK-8 assay was performed to detect the effect of different concentration of erastin on cell viability of HGC-27 cells. (B) The effect of $6.23 \mu \mathrm{M}$ of erastin on cell viability of HGC-27 cells. "P<0.05, vs. Mock group. (C) EdU staining was performed to image dividing cells. "P<0.05, vs. Mock group. (D) Cell cycle phases were analyzed by flow cytometry following PI staining. ${ }^{\mathrm{P}}<0.05$, vs. Mock group. EdU, 5-ethynyl-20-deoxyuridine; PI, propidium iodide.
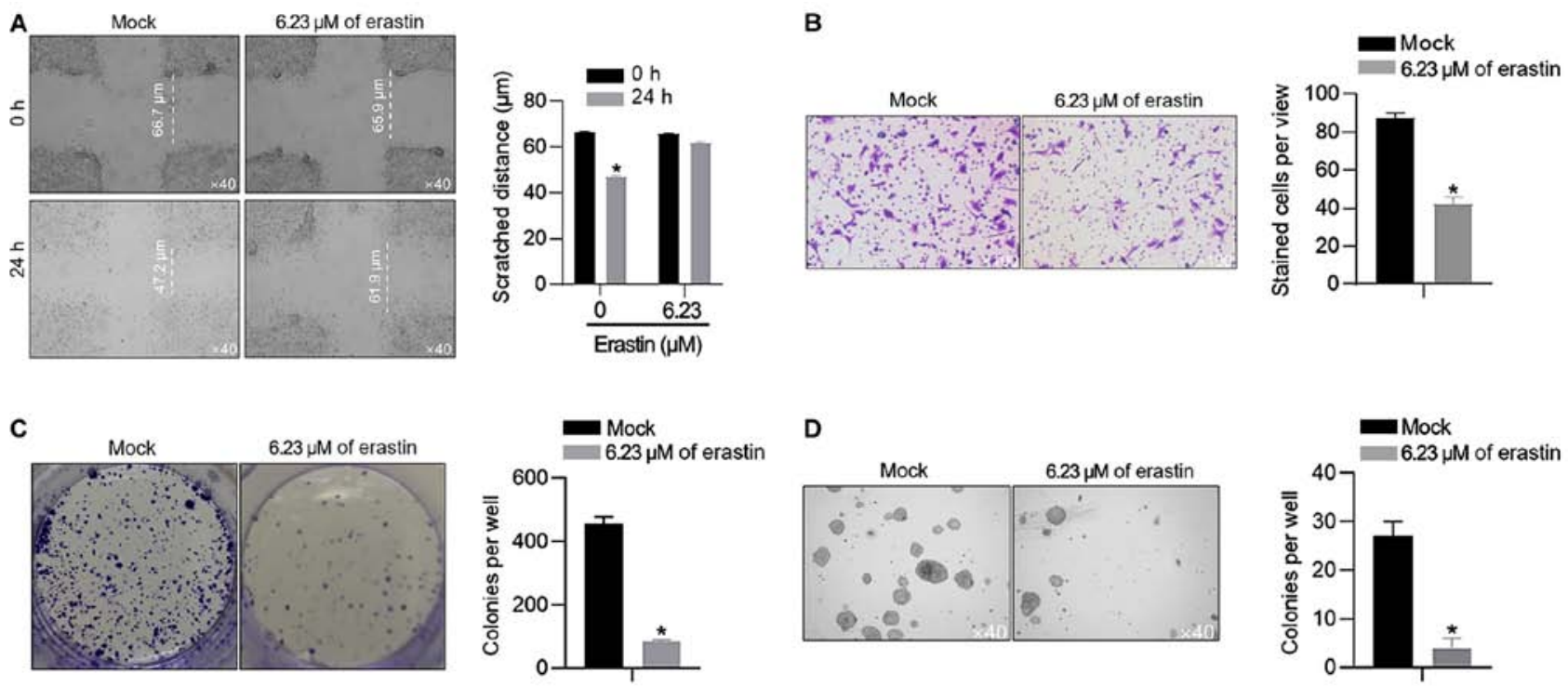

Figure 2. Effects of $6.23 \mu \mathrm{M}$ of erastin on malignant behaviors of HGC-27 cells. After $6.23 \mu \mathrm{M}$ of erastin concentration treatment, the malignant behaviors of HGC-27 were analyzed, including (A) migration, ${ }^{~} \mathrm{P}<0.05$, vs. 0 h group, (B) invasion, (C) colony formation and (D) tumor formation in soft agar, ${ }^{~} \mathrm{P}<0.05$, vs. Mock group. 

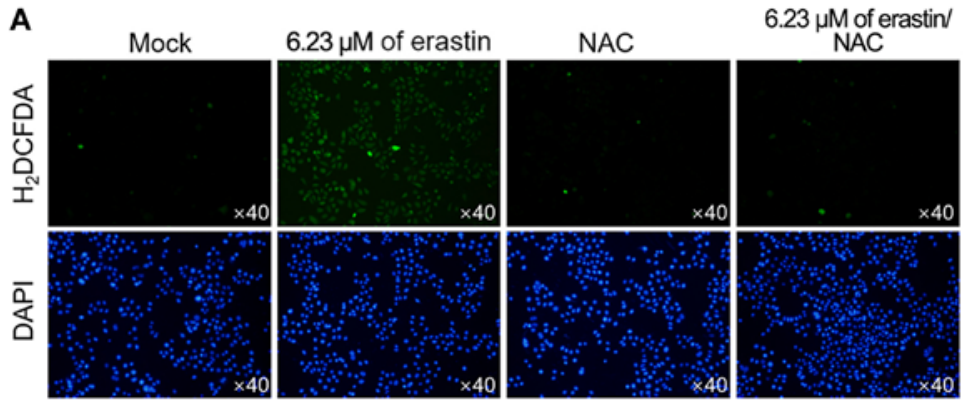

B

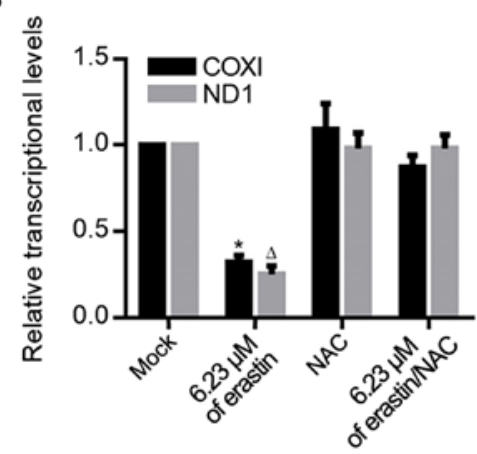

C

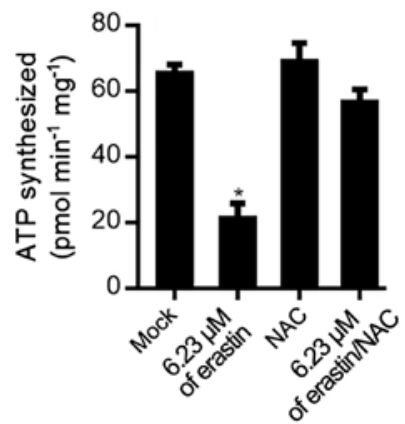

D
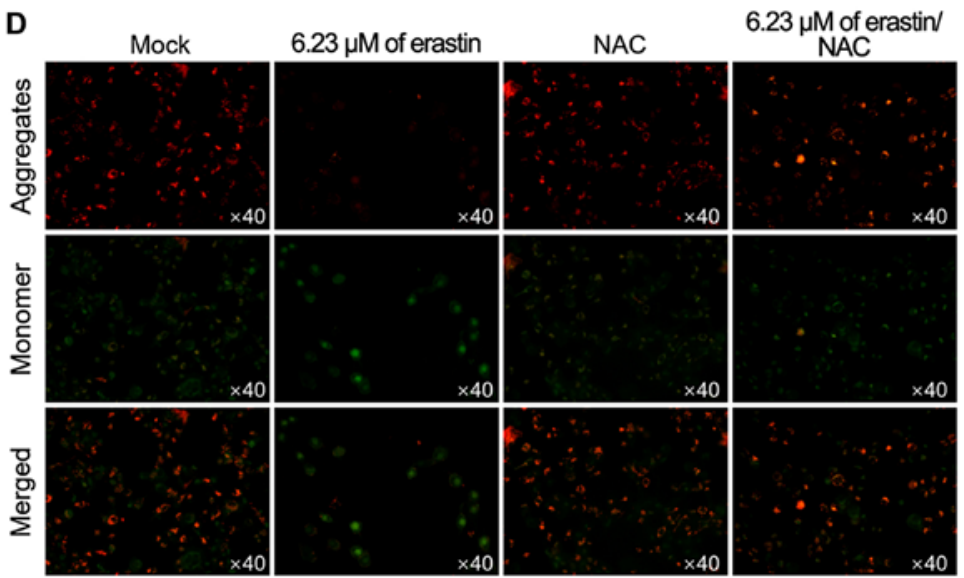

Figure 3. Erastin treatment induced ROS accumulation and mitochondrial dysfunction. (A) ROS detection was performed after erastin treatment. For measuring the effects of erastin on mitochondria (B) transcriptional activity of mitochondria ( $\mathrm{P}<0.05$ vs. COXI amount in Mock group; ${ }^{\wedge} \mathrm{P}<0.05$ vs. NDI amount in Mock group) and (C) ATP production activity of mitochondria ( ${ }^{*} \mathrm{P}<0.05$, vs. Mock group) were measured after 24-h treatment. (D) JC-1 staining was performed after erastin treatment for $24 \mathrm{~h}$. ROS, reactive oxygen species.

increase of positive signal, which was abolished by the addition of $10 \mu \mathrm{M}$ NAC, a ROS scavenger. ROS is a critical factor in the maintenance of mitochondrial homeostasis, thus mitochondrial function was measured by transcriptional activity and ATP production. As shown in Fig. 3B, erastin treatment significantly decreased transcripts COXI and ND1 in mitochondria and decreased the amount of ATP synthesized (Fig. 3C), demonstrating that $6.23 \mu \mathrm{M}$ of erastin clearly caused mitochondrial dysfunction. Accumulated ROS modulates mitochondrial function mainly by regulating mitochondrial potential, so mitochondrial potential was detected following erastin treatment by JC-1 staining. As shown in Fig. 3D, erastin treatment clearly decreased JC-1 aggregates and increased JC-1 monomers and this was reversed by $10 \mu \mathrm{M}$ NAC treatment.

Long-term treatment of low dose of erastin induces apoptosis, but not ferroptosis, and results in ROS accumulation.
As shown above, $6.23 \mu \mathrm{M}$ of erastin inhibited proliferation from 1-5 days (Fig. 1) potentially by causing mitochondrial dysfunction. By hypothesizing that mitochondrial dysfunction may cause oxidative stress and subsequently lead to cell death, whether $6.23 \mu \mathrm{M}$ of erastin treatment causes cell death in long term was investigated. Cells treated with $6.23 \mu \mathrm{M}$ of erastin for 7 days were analyzed by CFSE/PI double staining. As shown in Fig. 4A, erastin evidently increased CFSE/PI double-stained cells, which was reversed by apoptosis inhibitor, zVAD and ROS scavenger NAC, but not by ferroptosis inhibitor ferrostatin-1, indicating that erastin-induced cell death occurred mainly by inducing apoptosis, but not by inducing ferroptosis. For specific analysis of apoptosis, annexin V/PI double staining was performed and the results showed that erastin treatment significantly increased proportion of Annexin V+/PI- and Annexin V+/PI+, which was reversed by zVAD or NAC co-treatment, but not by ferrostatin-1 co-treatment 
A

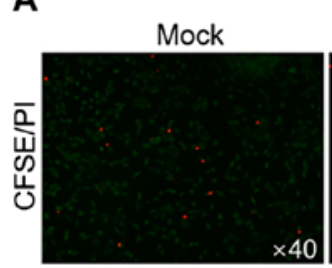

B
Erastin

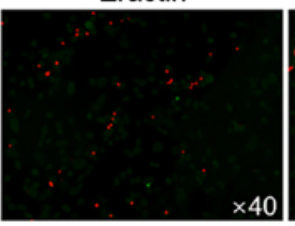

Erastin/

Ferrostain-1

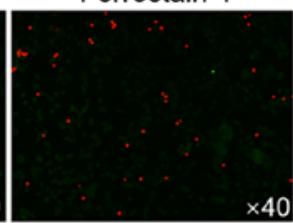

Erastin/

ZVAD

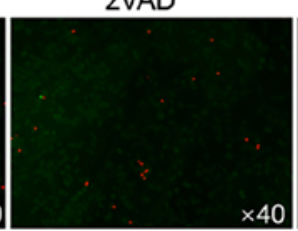

Erastin/

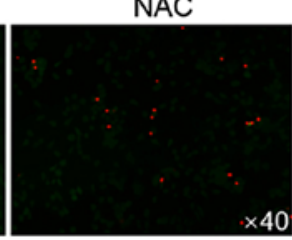

Erastin/ zVAD

Erastin/

NAC
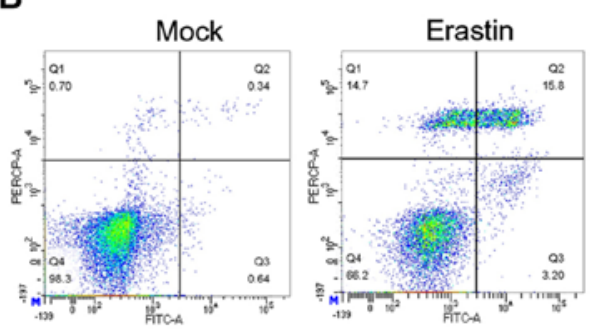

Erastin/
Ferrostain-1
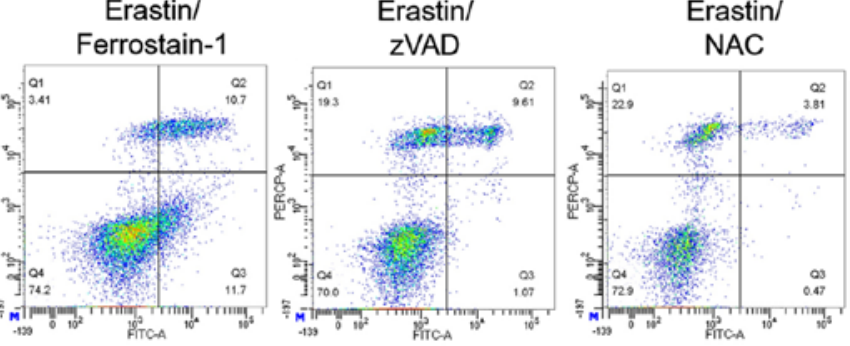

C

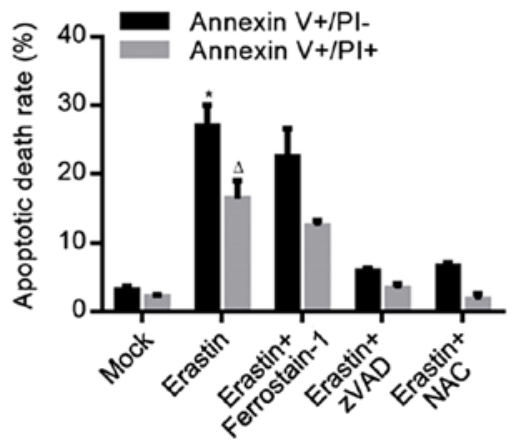

D

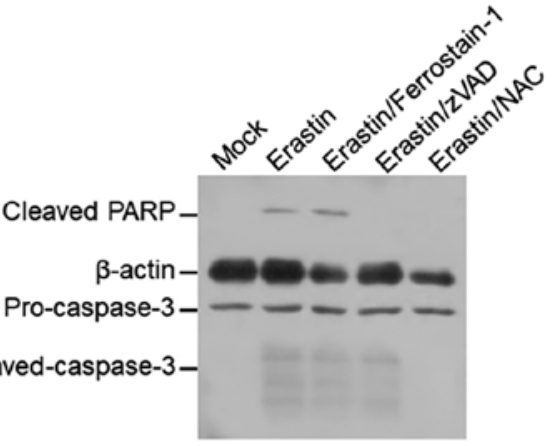

Figure 4. Effects of erastin on cell death in long-term treatment. (A) CFSE/PI double staining was performed to detect cell death after erastin treatment for 7 days. (B) Annexin V-FITC/PI double staining was performed to detect cell apoptosis after erastin treatment for 7 days. (C) Statistical analysis of cell apoptosis was performed. ${ }^{*} \mathrm{P}<0.05,{ }^{\Delta} \mathrm{P}<0.05$, vs. Mock group. (D) Western blot was performed to detect apoptotic-specific hallmarks, including cleaved PARP and cleaved caspase-3. CFSE, carboxyfluorescein diacetate, succinimidyl ester; PI, propidium iodide; PARP, poly (adenosine diphosphate-ribose) polymerase.

(Fig. 4B and C). To further confirm apoptosis induced by erastin, apoptotic hallmarks including cleaved PARP and cleaved caspase-3 were imaged by western blot and consistent with previous results, erastin treatment evidently increased cleaved PARP and cleaved caspase-3 (Fig. 4D).

\section{Discussion}

Erastin was first revealed as a small molecule compound that specifically targets human tumor cells and induces non-apoptotic cell death without affecting the isogenic normal cell counterparts (14). The non-apoptotic cell death induced by erastin was later identified as ferroptosis, which is a novel form of cell death that is dependent on the regulation of iron (15). Researchers have also shown that erastin-induced ferroptosis occurs mainly by inhibiting the cystine/glutamate antiporter (system $\mathrm{X}_{\mathrm{C}}^{-}$), thus blocking the uptake of cystine and resulting in the accumulation of $\operatorname{ROS}(15,16)$. Considering the important dual effects of ROS in maintaining the homeostasis of physiological processes and causing oxidant stress, the accumulated ROS induced by erastin are thought to be closely associated with cell behavior.

The present study observed the effects of a relatively low dose of erastin on HGC-27 gastric cancer cells. To avoid inducing acute cell death in a ferroptotic manner, the HGC-27 cells were treated with a $30 \%$ inhibitory concentration $\left(\mathrm{IC}_{30}\right)$ of erastin. Treatment with the $6.23 \mu \mathrm{M}$ of erastin resulted in the accumulation of ROS and inhibition of cell proliferation by blocking the cell cycle at the G1/G0 phase. It also clearly inhibited the malignant behaviors of HGC-27 cells, including migration, invasion, colony formation and tumor formation in soft agar. It has been reported that the inhibition of cystine and decrease in GSH causes mitochondrial dysfunction by causing ROS accumulation $(17,18)$; thus, mitochondrial function was measured. Erastin treatment notably decreased the mitochondrial potential, transcriptional activity and ATP production, which were all restored by cotreatment with $10 \mu \mathrm{M}$ NAC, a ROS scavenger, demonstrating that the erastin-induced ROS accumulation markedly depolarized mitochondria and inhibited mitochondrial function. According to the results, ROS accumulation induced by erastin successfully induced mitochondrial dysfunction, although whether mitochondrial mass was affected was unknown $(17,18)$. It is worth investigating the mitochondrial mass by measuring mitochondrial genome following erastin treatment.

Erastin-induced ferroptosis has been widely studied; however, the mechanism of a relatively low dose of erastin treatment in the long term remains to be elucidated. To this end, HGC-27 cells were treated with the $6.23 \mu \mathrm{M}$ of erastin 
for 1, 3 and 7 days, and cell death was evaluated. As shown in the results, although there was no evident induction of death at 1 and 3 days (data not shown), erastin significantly induced cell death after 7 days of treatment, which was not reversed by ferrostatin-1, but was reversed by zVAD, a mitochondria-related apoptosis inhibitor and NAC, a ROS scavenger. Annexin V-PI double staining analysis by flow cytometry and detection of cleaved PARP and cleaved caspase-3, which are hallmarks of apoptosis, by western blotting further confirmed that long-term treatment with erastin mainly induced apoptosis. Although the results focused on apoptosis and failed to determine whether long-term treatment with a low dose of erastin induced ferroptosis, the addition of the ferrostatin-1 failed to clearly affect cell death, indicating the slight effect of low-dose erastin treatment on inducing ferroptosis.

In summary, the present study determined that low-dose erastin treatment resulted in ROS accumulation and thus induced mitochondrial dysfunction. It was also observed that low-dose erastin treatment decreased the malignant behaviors of HGC-27 cells in the short term and mainly induced apoptosis, but not ferroptosis, in the long term. These results may provide an additional understanding of the antitumor effects of erastin.

\section{Acknowledgements}

The authors wish to thank Dr Huimin Shi (Sichuan University) for language editing.

\section{Funding}

The present study was supported by Scientific Research Startup Fund for Scholars, Medicine School of Medical and Life Sciences, Chengdu University of Traditional Chinese (grant no. 18110) and Scientific Fund of the Chengdu University of Traditional Chinese Medicine (grant no. 077035012).

\section{Availability of data and materials}

The datasets used and/or analyzed during the current study are available from the corresponding author on reasonable request.

\section{Authors' contributions}

YS, RD and CZ contributed to conception, design and acquisition of data. CZ contributed to acquisition, analysis and interpretation of data. YS, RD and CZ contributed to cell culture. All authors read and approved the final manuscript.

\section{Ethics approval and consent to participate}

Not applicable.

\section{Patient consent for publication}

Not applicable.

\section{Competing interests}

The authors declare that they have no competing interests.

\section{References}

1. Xie Y, Hou W, Song X, Yu Y, Huang J, Sun X, Kang R and Tang D: Ferroptosis: Process and function. Cell Death Differ 23: 369-379, 2016.

2. Yang WS, SriRamaratnam R, Welsch ME, Shimada K, Skouta R, Viswanathan VS, Cheah JH, Clemons PA, Shamji AF, Clish CB, et al: Regulation of ferroptotic cancer cell death by GPX4. Cell 156: 317-331, 2014.

3. DeHart DN, Fang D, Heslop K, Li L, Lemasters JJ and Maldonado EN: Opening of voltage dependent anion channels promotes reactive oxygen species generation, mitochondrial dysfunction and cell death in cancer cells. Biochem Pharmacol 148: 155-162, 2018

4. Pan X, Lin Z, Jiang D, Yu Y, Yang D, Zhou H, Zhan D, Liu S, Peng G, Chen Z and Yu Z: Erastin decreases radioresistance of NSCLC cells partially by inducing GPX4-mediated ferroptosis. Oncol Lett 17: 3001-3008, 2019.

5. Schieber M and Chandel NS: ROS function in redox signaling and oxidative stress. Curr Biol 24: R453-R462, 2014

6. D'Autreaux B and Toledano MB: ROS as signalling molecules: Mechanisms that generate specificity in ROS homeostasis. Nat Rev Mol Cell Biol 8: 813-824, 2007.

7. Valko M,Leibfritz D, Moncol J, Cronin MT, Mazur M and Telser J: Free radicals and antioxidants in normal physiological functions and human disease. Int J Biochem Cell Biol 39: 44-84, 2007.

8. Trachootham D, Alexandre J and Huang P: Targeting cancer cells by ROS-mediated mechanisms: A radical therapeutic approach? Nat Rev Drug Discov 8: 579-591, 2009.

9. Ferlay J, Shin HR, Bray F, Forman D, Mathers C and Parkin DM: Estimates of worldwide burden of cancer in 2008: GLOBOCAN 2008. Int J Cancer 127: 2893-2917, 2010.

10. Wagner AD, Grothe W, Haerting J, Kleber G, Grothey A and Fleig WE: Chemotherapy in advanced gastric cancer: A systematic review and meta-analysis based on aggregate data. J Clin Oncol 24: 2903-2909, 2006.

11. Power DG, Kelsen DP and Shah MA: Advanced gastric cancer-slow but steady progress. Cancer Treat Rev 36: 384-392, 2010.

12. Chen W, Zou P, Zhao Z, Chen X, Fan X, Vinothkumar R, Cui R, Wu F, Zhang Q, Liang G and Ji J: Synergistic antitumor activity of rapamycin and EF24 via increasing ROS for the treatment of gastric cancer. Redox Biol 10: 78-89, 2016.

13. Livak KJ and Schmittgen TD: Analysis of relative gene expression data using real-time quantitative PCR and the 2(-Delta Delta C(T)) method. Methods 25: 402-408, 2001.

14. Dolma S, Lessnick SL, Hahn WC and Stockwell BR: Identification of genotype-selective antitumor agents using synthetic lethal chemical screening in engineered human tumor cells. Cancer Cell 3: 285-296, 2003.

15. Dixon SJ, Lemberg KM, Lamprecht MR, Skouta R, Zaitsev EM, Gleason CE, Patel DN, Bauer AJ, Cantley AM, Yang WS, et al: Ferroptosis: An iron-dependent form of nonapoptotic cell death. Cell 149: 1060-1072, 2012.

16. Dixon SJ, Patel DN, Welsch M, Skouta R, Lee ED, Hayano M, Thomas AG, Gleason CE, Tatonetti NP, Slusher BS and Stockwell BR: Pharmacological inhibition of cystine-glutamate exchange induces endoplasmic reticulum stress and ferroptosis. Elife 3: e2523, 2014.

17. Diotte NM, Xiong Y, Gao J, Chua BH and Ho YS: Attenuation of doxorubicin-induced cardiac injury by mitochondrial glutaredoxin 2. Biochim Biophys Acta 1793: 427-438, 2009.

18. Wang J, Pan S and Berk BC: Glutaredoxin mediates Akt and eNOS activation by flow in a glutathione reductase-dependent manner. Arterioscler Thromb Vasc Biol 27: 1283-1288, 2007. 